(2) Open Access Full Text Article

ORIGINALRESEARCH

\title{
Analysis of the Public Health Functions of the Chinese Government in the Prevention and Control of COVID-19
}

This article was published in the following Dove Press journal: Risk Management and Healthcare Policy

\section{Pengfei Zhang}

School of Labor and Human Resources, Renmin University of China, Beijing, People's Republic of China
Correspondence: Pengfei Zhang School of Labor and Human Resources, No. 59, Zhongguancun Street, Beijing 100872, People's Republic of China Email pfzoffice@ruc.edu.cn
Background: The focus of the study is to assess the defects of the public health functions of the Chinese government in the prevention and control of COVID-19 and to explore ways to improve the public health functions of the Chinese government.

Methods: This study adopted a qualitative design. A logical framework based on the theoretical underpinning of public health functions of the government in the prevention and control of a major public health crisis was constructed. We compared the public health functions of the Chinese government and the effectiveness of these functions for COVID-19. Results: The defects of the public health functions of the Chinese government in the prevention and control of COVID-19 include a lack of crisis prediction, initiative and timeliness in crisis early warning functions; weak crisis security functions; and a lack of functions for handling the aftermath. The Chinese government should improve its crisis early warning functions, strengthen its crisis security functions, and establish functions for handling the aftermath.

Conclusion: The defects of the public health functions of the Chinese government in the prevention and control of COVID-19 can provide referential value for other countries. Especially, by continuously improving the construction of standard pandemic early warning procedures and regulations, optimizing the pandemic treatment system, and strengthening the fairness and accessibility of medical services, countries with severe pandemic situations may be able to reduce the impact of COVID-19 on public health more efficiently.

Keywords: COVID-19, public health functions, prevention and control, defects

\section{Introduction}

Improving the public health functions of governments and strengthening their ability to respond to major public health crises are important means to ensure the health of citizens. The public health functions of a government reflect the government's role and responsibilities in the prevention and control of major public health crises. First, due to the systematic and pluralistic characteristics of a major public health crisis, it is necessary for governments to integrate all aspects of public power in the prevention and control process and promote and coordinate the close cooperation between regional prevention and control departments and local residents. This is because relying only on regional prevention and control departments or relying only on local residents cannot fundamentally eliminate the crisis. Therefore, governments should play a role in controlling the overall situation when handling major public health crises. Second, due to the public characteristics 
of major public health crises, it is necessary to formulate targeted public policies in the prevention and control process to guide and regulate the production and living behaviors of citizens. Therefore, governments should bear the main responsibility in handling major public health crises. In addition, regarding the quasi-public attribute of medical services, governments also needs to provide medical services efficiently through well-established public health functions after the emergence of a major public health crisis. In particular, it is necessary for governments to maintain the level of medical service supplies and effectively ensure the fairness and accessibility of medical services. It can be seen that the public health functions of governments are highly related to the prevention and control of major public health crises, and the perfection and implementation of the functions determine the impact of major public health crises.

Since the outbreak of COVID-19, the virus's pathological characteristics, such as its high infectivity, long incubation period, wide population susceptibility and high fatality rate, have brought severe challenges to pandemic prevention and control worldwide. Especially with the internationalization of the mobile population and economic globalization, the spread of COVID-19 around the world has become easier, which has brought serious social and economic impacts to countries with imperfect public health functions. The Chinese government has been focusing on improving public health functions since 2017, putting the prevention and control of major public health crises on the list of the priority government functions. ${ }^{1}$ In the COVID-19 prevention and control process, the Chinese government is committed to highly integrating COVID-19 prevention and control with its public health functions. By formulating temporary policies, the Chinese government has concentrated the capabilities of medical institutions in various regions to serve areas with severe pandemic situations, including limiting population mobility in areas with severe outbreaks ${ }^{2}$ and allocating more doctors, medical facilities and medical materials to areas with severe pandemic situations. ${ }^{3}$ Although the Chinese government has made some achievements in the prevention and control of COVID-19, the public health functions of the Chinese government still have defects, and these defects need to be alerted by other countries. Especially for countries with severe COVID-19 situations, while learning from China's successful experience, they should also avoid defects in the public health functions of the Chinese government, which may help them better formulate prevention and control policies for COVID-19. Therefore, this article studies the defects in the public health functions of the Chinese government in the prevention and control of COVID-19.

\section{Methods}

This study adopted a qualitative design. A logical framework based on the theoretical underpinning of public health functions of the government in the prevention and control of a major public health crisis was constructed. Through the framework we compared the public health functions of the Chinese government and the effectiveness of these functions for COVID-19.

The development of a major public health crisis can be divided into three stages: the ex-ante stage, the interim stage and the ex-post stage. ${ }^{4,5}$ The ex-ante stage is a kind of advanced control of a major public health crisis. In the ex-ante stage, the purpose is not only to control the crisis within a certain range and prevent its spread, but also to study the causes of the crisis and prevent it at its roots. Due to the small scale and strong controllability of a major public health crisis, the ex-ante stage is the best stage to resolve the crisis. ${ }^{6}$ However, a major public health crisis usually has no obvious signs in the ex-ante stage, making it difficult to detect the crisis in time. The interim stage is the stage in which a major public health crisis spreads and begins to have great impacts. After entering this stage, the crisis begins to have wide impacts on national economic development, the social order and people's health. The interim stage is the main stage in which the public health functions of governments play a role in the prevention and control of major public health crises. In the interim stage, the purpose is to control the spread of a crisis and provide effective solutions to the social groups damaged by the crisis. ${ }^{7}$ As time passes, various characteristics of a major public health crisis are gradually exposed, and the government, though its public health functions, can implement targeted prevention and control measures based on the characteristics of the crisis. Establishing a security system that meets the public health needs during a major public health crisis, guiding the public to cooperate with prevention and control departments, increasing the use of prevention and control experience and increasing the use of the knowledge learned from successful cases are important means to prevent the further expansion of a crisis in the interim stage. The ex-post stage is a process in which the crisis is completely controlled and gradually eliminated. ${ }^{8}$ In the ex-post stage, first, it is necessary to restore 
economic development and the social order as soon as possible to ensure the normal production and lives of the public. Second, it is also necessary to evaluate and summarize the major public health crisis, preserve the successful prevention and control experience, and improve the deficiencies in the prevention and control process. The expost stage is the best time to transform the implicit prevention and control experience into explicit knowledge. Through investigation, evaluation, case studies, and classification, the knowledge database of major public health crises is enriched so as to provide abundant experience for the prevention and control of major public health crises in the future. In different stages of a major public health crisis, there are significant differences in the public health functions of governments.

\section{Ex-Ante Stage: Crisis Early Warning Functions}

A crisis early warning means that the government actively detects the signs that may trigger a major public health crisis; and through information collection, information analysis, crisis prediction and crisis pre-control procedures, timely prevention measures can be formulated to avoid the spread of the crisis. ${ }^{9,10}$ Regarding information collection, the public health function of the government is to establish a sensitive and accurate public health information monitoring system, strengthen the construction of public health monitoring facilities and the technical level of monitoring personnel, establish warning indicators for factors that may trigger crises, and closely monitor the change signals of factors that may trigger crises so as to comprehensively grasp the development trend of the situation. In terms of information analysis, the public health function of governments is to classify and sort public health information, screen out information that may trigger crises, process the information through scientific means, and form an information database and case database to provide a comprehensive scientific basis for subsequent crisis prediction. Regarding crisis prediction, the public health functions of governments are to predict the severity, evolutionary trend and impact scope of a crisis according to the characteristics of the crisis obtained from information analysis and update and maintain the crisis prediction results in a timely manner as the crisis develops. Crisis prediction is error-prone in the ex-ante stage. First, due to the existence of subjectivity in the crisis information collection process, crisis information analysis may not be comprehensive, resulting in failing to discover a crisis in time and missing the best period for the prevention and control of the crisis. Second, because the development of a crisis objectively includes a large number of uncertain factors, it is easy to misjudge the spread speed and impact scope of the crisis, resulting in misleading the follow-up crisis pre-control operations. Regarding crisis pre-control, based on the development trend of a crisis, the public health function of governments is to formulate emergency plans and policies to eliminate the crisis in its infancy as much as possible. This process requires a high degree of combination of pre-control operations and crisis prediction. Through the accurate grasp of crisis prediction, governments should scientifically formulate response plans, efficiently coordinate medical resources and personnel scheduling, and disclose crisis information to the public in a timely manner. The success or failure of pre-control determines whether the follow-up prevention and control work of the crisis needs to be further conducted. ${ }^{11}$ The success of pre-control means that the crisis is eliminated in its infancy, avoiding huge economic and social stability losses. The failure of pre-control means that a crisis has spread and the prevention and control of the crisis has entered the interim stage.

\section{Interim Stage: Crisis Security Functions}

Crisis security refers to the set of policies, measures and methods formulated by a government to conduct its responsibility for the prevention and control of major public health crises in the interim stage. ${ }^{12}$ The primary crisis security function of a government is to strengthen the guidance and regulation of public behavior in the interim stage. Because of the complex characteristics of a major public health crisis, the source of infection is difficult to detect and the transmission route is hardly observed in the short term, resulting in the spread of the crisis being closely related to the self-prevention behavior of the public. Only when the public has comprehensive crisis awareness can they effectively establish scientific preventive measures in complex production and life and improve their abilities to respond to the crisis. Therefore, first, the crisis security function of governments is to strengthen the guidance and regulation of the public behavior to ensure that the public has scientific and comprehensive awareness of a major public health crisis. Second, the crisis security of governments should promote cooperation among prevention and control departments. In the interim stage, a major public health crisis begins to spread 
and gradually causes damage. Therefore, the crisis prevention and control work involves the interwoven cooperation of multiple government departments. ${ }^{13}$ However, the unique functional structure among government departments hinders the cooperation of prevention and control work. The division of functions between government departments can easily lead to overlapping and unclear responsibilities for crisis prevention and control work, resulting in a slow response capacity to crises and confusion regarding crisis management systems. Therefore, it is necessary for crisis security to strengthen the cooperation among prevention and control departments and improve the efficiency of prevention and control work. Third, the crisis security of governments should ensure the accessibility of medical services to the citizens affected by a crisis. A major public health crisis usually has serious impacts on public health. People affected by crises need to be treated systematically, and their treatment costs are relatively high, resulting in poor accessibility to medical services for the population affected by the crises. ${ }^{14,15}$ Therefore, while paying attention to prevention and control work in the interim stage, governments should ensure that cases affected by crises receive timely and effective treatment. Fourth, the crisis security of governments should ensure sufficient medical resources. Many medical resources will be used in the prevention, control and treatment process of a major public health crisis, and sufficient supplies of medical materials, treatment facilities, and medical personnel are the basis of the process. Especially for the regions seriously affected by a crisis, the supply of medical resources is seriously in conflict with the demand. Therefore, governments should ensure that medical resources can flow freely among regions and especially promote more medical resources to flow to the regions seriously affected by a crisis.

\section{Ex-Post Stage: Functions of Handling the Aftermath}

The functions of handling the aftermath represent the set of systems formulated by governments to accelerate economic and social recovery and promote the efficient evaluation of the prevention and control work after a major public health crisis has been effectively controlled and gradually subsided. In the ex-post stage, the functions of handling the aftermath are mainly composed of the following three aspects: First, it is necessary for the government to restore economic development and the social order as soon as possible to ensure the normal production and lives of the public. ${ }^{16}$ Especially for the people and industries seriously affected by a crisis, governments should give them adequate support for policy and finance to encourage them to get back on track. Second, it is necessary to summarize the prevention and control experience. ${ }^{17}$ Governments should analyze successful cases and refine their prevention and control experience so as to provide guidance for unexpected crises that may occur in the future. Furthermore, the government should summarize and improve the shortcomings in their prevention and control work in a timely manner to avoid repeating the same mistakes in the future. Third, governments need to reward relief managers or hold them accountable based on their performance and promptly disclose the results to the public. Reward and accountability are closely related to the restoration of social trust, and timely reward and accountability can reduce the negative impacts of a major public health crisis on social trust.

Based on the above theoretical underpinning and analysis, the logical framework of the public health functions of governments in the prevention and control of major public health crises is shown in Figure 1.

\section{Results}

COVID-19 is a major public health crisis that has had serious impacts on China's economic development, social order and people's health. As the main body of prevention and control of COVID-19, the Chinese government plays a major role in the research and judgment of pandemic information, the investigation and diagnosis of pandemic situations, and the coordination of medical supplies and treatment. However, the prevention and control process also exposes some defects in the public health functions of the Chinese government. Based on the theoretical underpinning of the public health functions of the government in the prevention and control of major public health crises, the defects of the public health functions of the Chinese government in the prevention and control of COVID-19 are mainly manifested in the following aspects.

\section{The Lack of Crisis Prediction, Initiative and Timeliness in Crisis Early Warning Functions}

The public health functions of the Chinese government in the prevention and control of COVID-19 lack crisis prediction. The key to preventing the spread of COVID-19 is to conduct 


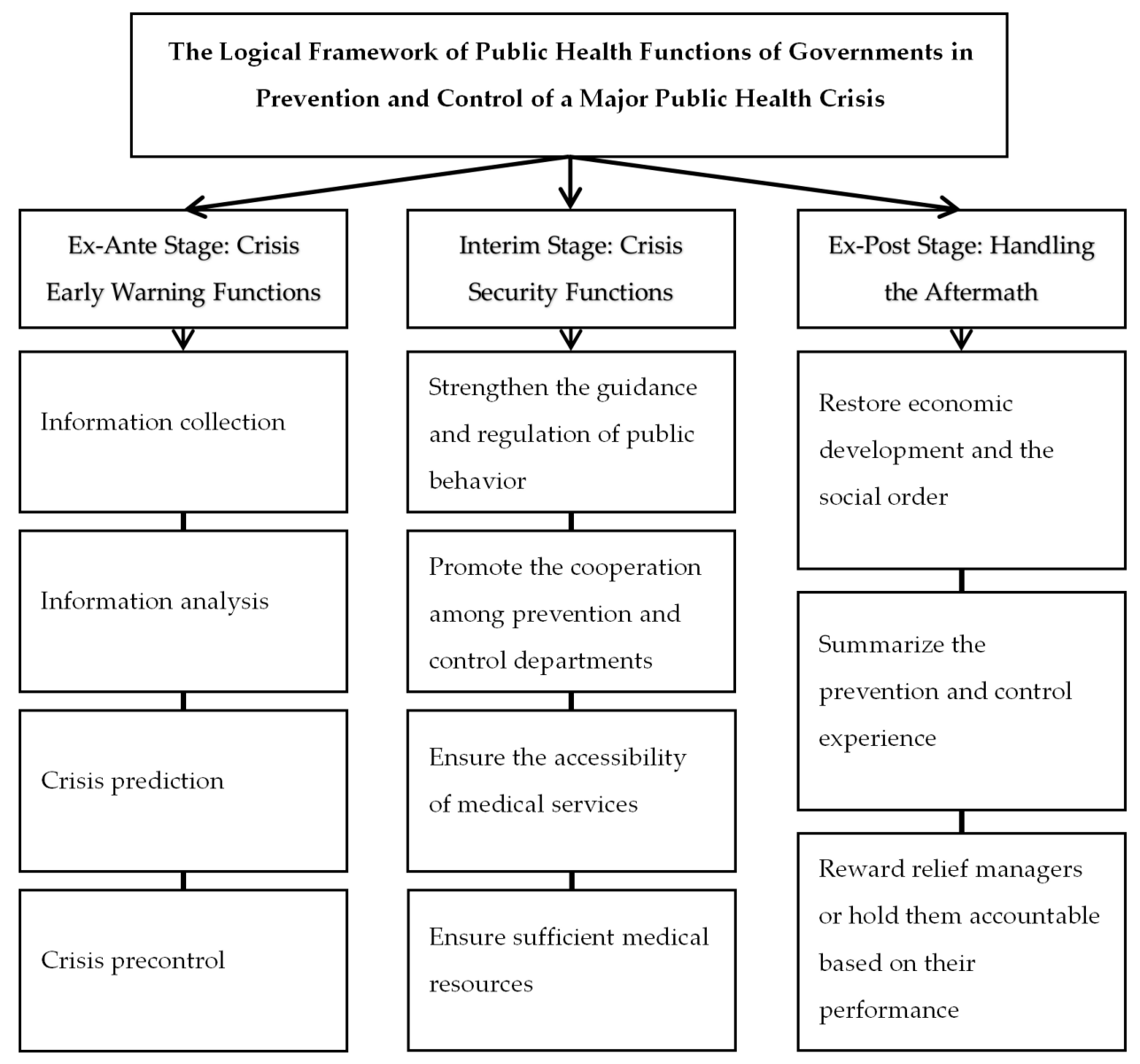

Figure I The logical framework of the public health functions of governments in the prevention and control of a major public health crisis.

sufficient crisis prediction. This requires that every crisis early warning process must be established "in advance" so as to achieve advanced information collection feedback, advanced crisis prediction update and maintenance, and advanced prevention and control intervention. Sufficient crisis prediction can buy time for the government's prevention and control work to slow down the spread of COVID-19. In the pandemic prevention and control process, the Chinese government has not specialized in collecting and analyzing crisis information, and the efficiency of collecting and analyzing information is low, resulting in slow crisis pre-control work. For example, there were signs of COVID-19 in China in December 2019, but due to the lack of crisis prediction, ${ }^{18}$ the actual time when formal prevention and control measures were taken was January $22,2020 .{ }^{19}$ It took nearly one month from the discovery of COVID-19 to crisis pre-control, resulting in the rapid spread of the pandemic.

There is also a lack of initiative in crisis early warning function. Crisis early warning emphasizes that the attitude towards a crisis changes from "passive handling" to "active monitoring", and the prerequisite for crisis early warning operations is to actively detect a crisis rather than passively waiting for a crisis to occur. The various characteristics of the major public health crisis in the ex-ante stage are recessive and can only be detected actively. Once the characteristics of a crisis are fully revealed, it usually means that the crisis has begun to break out. Therefore, passively waiting for the occurrence of a crisis means that the best time for prevention and control work is missed. For the Chinese government, the prevention and control of COVID-19 is a passive process. This is because the Chinese government's awareness of the pandemic is relatively weak, and the government has not actively monitored pandemic information, resulting in failure to take measures at the best time for the prevention and control of COVID-19. When COVID-19 spread and had a serious impact on public health, only a series of passive measures could be adopted for the prevention and control of COVID-19. For example, the Chinese government decided to lock down the city of Wuhan on January $23,2020,{ }^{20}$ 
and 25 regions in China announced the activation of a first-level public health emergency response on January 25, 2020. ${ }^{21}$ These were all passive measures taken because of the rapid spread of COVID-19.

Furthermore, the crisis early warning public health functions of the Chinese government lack timeliness. The emergence and variability of a major public health crisis require that crisis early warning should be timely. First, combined with crisis prediction, the government should make timely judgments and preventive decisions on complex crisis information. Second, it is necessary for the government to transform preventive decisions into action according to standard procedures, conduct timely precontrol, improve the prevention execution efficiency, and restrain the further development of the crisis. The government should also pay attention to the information disclosure timeliness and objectively disclose crisis information and preventive decisions to the public. However, the Chinese government was not able to make timely decisions and pre-control and failed to make the pandemic information available to the public in a timely and objective manner, resulting in the spread of COVID-19 and increasing the time costs and social costs of the prevention and control of COVID-19. For example, after the Chinese government discovered signs of the pandemic in December 2019, specific information on the pandemic and related preventive decisions were officially announced in January 2020 . $^{22,23}$

\section{Weak Crisis Security Functions}

First, the Chinese government has a weak crisis security legislation system. First, it is necessary to clarify the prevention and control obligations and responsibilities of the public and guide and regulate the public's production and life behaviors in accordance with the policies and regulations. Second, it is necessary to formulate a detailed legal basis and standard procedures for the crisis management process and clarify the duties performed by the various government departments in the crisis control process. To fulfill these responsibilities, it is necessary to strengthen the legal basis of prevention and control work to ensure efficient cooperation between various government departments. Furthermore, attention should be paid to operability to ensure that the duties performed by government departments are highly compatible with their responsibilities. The Chinese government lacks strong laws and regulations related to the prevention and control of COVID-19. For example, the lack of a standardized and timely emergency legislation system and the lack of a standardized treatment basis in the prevention and control of COVID-19 led to the failure to guide and regulate the public's production and life behaviors, resulting in an aggravation of the risk of cross infection. ${ }^{24}$ In addition, there are overlapping and uncoordinated responsibility systems among Chinese government departments, which leads to poor communication in the management of COVID-19 and low efficiency of pandemic prevention and control. ${ }^{25}$

Second, the Chinese government has a weak treatment system for COVID-19. During the spread of COVID-19, it was necessary to establish a centralized and efficient treatment command system to ensure that the treatment information was unobstructed, systematic and effective. In particular, the Chinese government should improve the treatment information network to realize the symmetry of information between the treatment institutions and the health administration departments, pay more attention to the timely mutual transformation of disease research and clinical treatment in the treatment process through the theoretical knowledge of disease research and practical experience in clinical treatment, make full use of modern advanced medical technology and strengthen international cooperation in treatment to compensate for shortcomings and improve the quality of COVID-19 treatment. However, there was asymmetric command information and delayed information feedback between the central government and local governments during the prevention and control of COVID-19. For example, the feedback efficiency of local governments in response to the macrolayout of prevention and control of COVID-19 is low, resulting in the central government being forced to supervise the behaviors of local governments through an inspection system. ${ }^{26}$ Moreover, due to the strong professional restrictions between the treatment institutions and the health administrative departments, the information communication in the treatment process is not smooth, and it is difficult for the health administrative departments to achieve accurate analysis of COVID-19. Therefore, it is difficult for the senior government to grasp the treatment information of COVID-19 in real time. For example, in order to obtain timely treatment information, the senior government directly calculates data statistics on suspected infected people through official websites. $^{27}$

Third, the Chinese government has a weak emergency medical system for COVID-19. A strong emergency medical system is conducive to improving the accessibility of 
medical services for the people affected by the crisis and ensuring that they can receive timely and effective treatment. A strong emergency medical system can put special drugs for the pandemic onto the drug lists of medical insurance. Through the drug lists of medical insurance, part of the costs of special drugs for the pandemic can be exempted, which can effectively reduce the burden of drug costs for patients and ensure the fairness of medical services. In addition, because cooperation among different regions is needed in the interim stage, the Chinese government needs to promote the harmonization of medical insurance systems among different regions. Therefore, a strong emergency medical system needs to unify medical insurance information between various regions and eliminate regional barriers. However, the Chinese government did not put special drugs for COVID-19 onto the drug lists of medical insurance until August 18, 2020, ${ }^{28}$ resulting in the poor accessibility of medical services for the people affected by COVID-19. Furthermore, medical institutions have serious overcrowding in areas with severe pandemic situations, ${ }^{29}$ resulting in an increased risk of cross infection. Moreover, there are great differences in the medical insurance systems among different regions in China, but the emergency medical system for the prevention and control of COVID-19 was not unified among regions until April 30, 2020. ${ }^{30}$

Fourth, the Chinese government has a weak reserve medical resource system. The sufficient supply of medical resources is the material basis for the prevention and control of COVID-19. This requires the Chinese government to reserve emergency medical resources to ensure that basic medical facilities, medical personnel and medical drugs are sufficient during the crisis. In order to effectively solve the inconsistency in the distribution of medical resources among regions during the prevention and control of COVID-19, the Chinese central government must centrally manage and allocate emergency medical resources. When emergency medical resources are insufficient to cope with the prevention and control of COVID-19, the Chinese government should establish a procurement system for emergency medical resources to improve the supply of medical resources. However, the Chinese government did not provide sufficient emergency medical resources for the public and failed to use the emergency medical resource procurement system in time. There was a shortage of emergency medical resources in the areas with severe pandemic situations, which caused some patients to not be diagnosed and treated in time. ${ }^{31,32}$

\section{Lacks of Functions for Handling the Aftermath}

The public health functions of the Chinese government lack the functions of handling the aftermath. In the expost stage, the Chinese government needs to actively participate in the calculation and compensation of losses to restore the normal operations of various industries. By continuously tracking the follow-up development of COVID-19, the Chinese government should confirm whether there are repeated signs of the pandemic so as to efficiently cooperate with the restoration of economic development and the re-establishment of the social order. Moreover, the Chinese government needs to analyze the causes of the crisis in detail and explore the imperfections in its work to summarize the lessons and make improvements. In addition, through the objective evaluation of the performance of relief managers, the Chinese government should reward them or hold them accountable so as to stimulate their consciousness and enthusiasm for work. Although the Chinese government has proposed methods to handle the aftermath, the process and standards of the handling the aftermath are not clear, resulting in the inability to efficiently coordinate the recovery of economic development and the stability of the social order. ${ }^{33,34}$ Moreover, the Chinese government has not established a formal system of rewards and penalties, and it can only take temporary measures to reward relief managers or hold them accountable. ${ }^{35}$

\section{Discussion}

COVID-19 has not only affected the economic development, social order and public health of many countries in the world, but it has also caused severe challenges to the public health functions of governments. In particular, the increasing mobility of populations and the frequent exchange of information among countries provide objective conditions for the development and spread of COVID-19, resulting in the complexity and difficulty of the prevention and control of COVID-19. The Chinese government has successful experience in the prevention and control of COVID-19, and the experience has been used as a reference by other countries. However, we believe that some defects of the Chinese government in the prevention and control of COVID-19 should be considered in other countries. Especially for the public health functions of governments, other countries should fully understand the defects of the Chinese government's public health functions 
in the prevention and control of COVID-19 so that they can continuously improve their public health functions of governments based on these defects. Learning from defects is far more important than learning from success in the prevention and control of COVID-19.

Based on the theoretical underpinning of the public health functions of governments in prevention and control of major public health crises and combined with the practice of the public health functions of the Chinese government in prevention and control of COVID-19, we found the following defects in the public health functions of the Chinese government: a lack of crisis prediction, initiative and timeliness in crisis early warning functions; weak crisis security functions, and a lack of functions for handling the aftermath. In order to respond to COVID-19 more effectively, China should improve the public health functions of the government, and other countries can also learn from the improved measures.

The Chinese government should improve its crisis early warning functions. The main reason why the Chinese government lacks crisis prediction, initiative and timeliness as crisis early warning functions is that the standard crisis early warning procedures and regulations are imperfect. Therefore, it is necessary for the Chinese government to improve the construction of standard crisis early warning procedures and regulations. The Chinese government needs to pay more attention to clarifying the standard procedures for information collection, information analysis, crisis prediction and crisis pre-control in the ex-ante stage of a major public health crisis. Specifically, the Chinese government should increase the breadth and depth of information collection, improve the professional level of information analysis, ensure the comprehensiveness and accuracy of crisis prediction, and promote the timeliness and transparency of crisis control and crisis precontrol. Furthermore, the Chinese government needs to formulate standards to regulate the behavior of relief managers and increase their awareness of a major public health crisis. In this way, it can be ensured that crisis monitors can actively detect the signs of a crisis, crisis decision makers can take the initiative to make crisis decisions, and operators can implement crisis decisions in time.

The Chinese government also needs to strengthen its crisis security functions. First, through the improvement of laws and regulations, the responsibilities of government departments and the obligations of the public in the prevention and control process of a major public health crisis are clarified, the smooth and efficient coordination of information among crisis prevention and control departments is guaranteed, and production and life behaviors are guided so as to form an efficient crisis prevention and control layout dominated by government departments and supported by public participation. Second, it is necessary to improve the treatment system of crises. The treatment process must be centralized and unified by the central government, and local governments must fully implement and provide timely feedback on the command of the central government so as to ensure the systematicity and accuracy of the treatment process. Combined with theoretical research rules and clinical treatment experience, the treatment methods of a major public health crisis should be updated in time. Furthermore, it is necessary to improve the emergency medical system of a major public health crisis. During crisis prevention and control, the Chinese government needs to appropriately reduce the burden of public medical expenses from the emergency medical system so as to improve the fairness and accessibility of medical services. In addition, it is necessary to improve the reserve medical resource system. Through the reserve and procurement of medical resources, the supply capacity of medical resources can be significantly enhanced in crisis prevention and control.

Finally, the Chinese government must establish functions for handling the aftermath. First, the normal operations of the market should be maintained through fiscal and social policies, and the appropriate reduction or exemption of taxes should be given to enterprises to restore economic development and the social stability. Second, through the establishment of an information database and a case database, it will be easy to summarize the crisis prevention and control experience, deepen the awareness of a major public health crisis, and consolidate the achievements of crisis management. Furthermore, through the establishment of a formal system of rewards and penalties, relief managers will be rewarded and held accountable in time so as to stimulate their awareness of their responsibility.

\section{Conclusion}

Based on the theoretical underpinning of public health functions of governments in the prevention and control of major public health crises and combined with a series of measures of the Chinese government in the prevention and control of COVID-19, this article found the defects of the public health functions of the Chinese government in prevention and control of COVID-19, which have strong referential value for the pandemic prevention and control of other countries in the world. In the prevention and control process of COVID-19, other countries can consider improving their crisis early warning functions, strengthening their crisis security 
functions, and establishing functions for handling the aftermath. Especially for countries with severe pandemic situations, while learning from China's successful experience, they should also avoid the defects in the public health functions of the Chinese government. By continuously improving the construction of standard procedures and regulations of pandemic early warning, optimizing the pandemic treatment system, and strengthening the fairness and accessibility of medical services, they may be able to reduce the impact of COVID-19 on public health more efficiently.

\section{Acknowledgments}

The author would like to thank the National Social Science Fund of China for providing funding for this study, and the author would like to express my great thanks to Dr. Jinghua Gao for her contributions to this research.

\section{Author Contributions}

The author made substantial contributions to conception and design, acquisition of data, or analysis and interpretation of data; took part in drafting the article or revising it critically for important intellectual content; agreed to submit to the current journal; gave final approval of the version to be published; and agree to be accountable for all aspects of the work.

\section{Funding}

The study was funded by the National Social Science Fund of China(20AZD076).

\section{Disclosure}

The author declared no potential conflicts of interest.

\section{References}

1. http://www.gov.cn/zhuanti/19thcpc/. Accessed January 12, 2021.

2. http://www.hubei.gov.cn/zhuanti/2020/gzxxgzbd/qfqk/202001/ t20200125_2014893.shtml.

3. http://www.hubei.gov.cn/zhuanti/2020/gzxxgzbd/qfqk/202002/ t20200214_2027353.shtml.

4. Costa G, Marra M, Salmaso S. Health indicators in the time of crisis in Italy. Epidemiol Prev. 2012;36(6):337-366.

5. Dafuleya G. Social and emergency assistance ex-ante and during COVID-19 in the SADC region[J]. Int $J$ Community Social Development. 2020;2(2):251-268. doi:10.1177/2516602620936028

6. Persad GC. A conceptual framework for clearer ethical discussions about Covid-19 response. Am J Bioethics. 2020;20(7):98-101. doi:10.1080/15265161.2020.1779400

7. Liu Q, Zheng Z, Zheng J, et al. Health communication through news media during the early stage of the COVID-19 outbreak in China: digital topic modeling approach. J Med Internet Res. 2020;22(4): e19118. doi: $10.2196 / 19118$
8. Hemel DJ, Rodriguez DB. A public health framework for COVID-19 business liability. Northwestern Public Law Research Paper. 2020;20-10:20-25.

9. O'brien SP. Crisis early warning and decision support: contemporary approaches and thoughts on future research[J]. Int Studies Rev. 2010;12(1):87-104. doi:10.1111/j.1468-2486.2009.00914.x

10. Alessi L, Detken C. On policymakers' loss functions and the evaluation of early warning systems: comment. Econ Lett. 2014;124 (3):338-340. doi:10.1016/j.econlet.2014.06.015

11. Pinto A, Saeed M, Sakka HE, et al. Setting up an early warning system for epidemic-prone diseases in Darfur: a participative approach. Disasters. 2005;29(4):310-322. doi:10.1111/j.03613666.2005.00294.x

12. Jakovljevic M, Bjedov S, Jaksic N, et al. COVID-19 pandemia and public and global mental health from the perspective of global health security. Psychiatr Danub. 2020;32(1):6-14. doi:10.24869/ psyd.2020.6

13. Wang H, Cleary PD, Little J, et al. Communicating in a public health crisis. Lancet Digital Health. 2020;2(10):e503. doi:10.1016/S25897500(20)30197-7

14. Hick JL, Biddinger PD. Novel coronavirus and old lessons - preparing the health system for the pandemic. New England $J$ Med. 2020;382(20):e55. doi:10.1056/NEJMp2005118

15. Tulenko K, Vervoort D. Cracks in the system: the effects of the Coronavirus pandemic on public health systems. Am Rev Public Administration. 2020;50(6-7):455-466.

16. Bonaccorsi G, Pierri F, Cinelli M, et al. Economic and social consequences of human mobility restrictions under COVID-19. Proc Nat Acad Sci. 2020;117(27):15530-15535. doi:10.1073/pnas.2007658117

17. Buheji M, da Costa Cunha K, Beka G, et al. The extent of covid-19 pandemic socio-economic impact on global poverty. a global integrative multidisciplinary review. Am J Economics. 2020;10(4):213-224. doi:10.5923/j.economics.20201004.02

18. h t t p : // m. n e w s. c c t v. c o m/2019/12/31/ ARTIL1JxT1xYZ9uMgxwJRDmJ191231.shtml. Accessed January 12, 2021.

19. http://www.wuhan.gov.cn/zwgk/xxgk/zfwj/gfxwj/.202003/ t20200316_973437.shtml.

20. http://www.wuhan.gov.cn/sy/whyw/.202003/t20200316_960171. shtml.

21. https://china.huanqiu.com/article/9CaKrnKp4bG. Accessed January 12, 2021.

22. http://wjw.wuhan.gov.cn/gsgg/.202004/t20200430_1199589.shtml.

23. http://wjw.wuhan.gov.cn/gsgg/.202004/t20200430_1199591.shtml.

24. http://www.wuhan.gov.cn/sy/whyw/.202003/t20200316_959144. shtml.

25. http://www.gov.cn/xinwen/.2020-05/15/content_5511993.htm.

26. http://js.people.com.cn/n2/2020/0316/c360300-33878009.html. Accessed January 12, 2021.

27. http://zwfw.hubei.gov.cn/webview/yqzq/yqsb.html. Accessed January 12, 2021.

28. http://www.gov.cn/fuwu/.2020-08/18/content_5535560.htm.

29. http://www.hubei.gov.cn/zfwj/ezbf/.202009/t20200930_2938567. shtml.

30. http://www.nhsa.gov.cn/art/.2020/4/30/art_54_3089.html.

31. h t t p://www.nh c.gov.cn/xcs/fkdt/.202004/ 0f0d0c57f20e454499a67b43ff00b3d6.shtml.

32. h t t p : / / w w w.n h c.gov.cn/x c s/xwbd/. $202004 /$ 0145eb77758a4e9781270c3911550fd8.shtml.

33. http://www.gov.cn/premier/.2020-03/16/content_5492014.htm.

34. http://www.gov.cn/xinwen/.2020-03/27/content_5496366.htm.

35. http://www.gov.cn/xinwen/.2020-03/05/content_5487522.htm. 


\section{Publish your work in this journal}

Risk Management and Healthcare Policy is an international, peerreviewed, open access journal focusing on all aspects of public health, policy, and preventative measures to promote good health and improve morbidity and mortality in the population. The journal welcomes submitted papers covering original research, basic science, clinical \& epidemiological studies, reviews and evaluations, guidelines, expert opinion and commentary, case reports and extended reports. The manuscript management system is completely online and includes a very quick and fair peer-review system, which is all easy to use. Visit http://www.dovepress.com/testimonials.php to read real quotes from published authors. 